\title{
Kann eine Colitis ulcerosa ausheilen?
}

Frage: Gibt es Hinweise

in der Literatur, die auch die Möglichkeit einer

kompletten Remission der Erkrankung untersucht haben? Ab welchem schubfreien Zeitraum kann von einer kompletten Ausheilung ausgegangen werden, oder ist dies bei der Colitis ulcerosa so nicht möglich?

Sind komplette
Remissionen bzw. Ausheilungen bei der Colitis ulcerosa beobachtet worden und wie häufig sind diese zu finden? Wie wird eine vollständige Ausheilung der Colitis ulcerosa definiert, ist dabei auch wieder eine normalisierte histologische Architektur der Mukosa zu finden?

Dr. Martin Held: Es gibt milde Krankheitsverläufe, bei denen es zu wenig klinischen Beschwerden kommt. Eine komplette Ausheilung einer Colitis ulcerosa mit histologisch völlig unauf- fälliger Schleimhaut ist meines Wisssens nicht beschrieben. Man muss hier meist die Erstdiagnose in Frage stellen. Die Erkrankung beschriebt ja eine chronisch entzündliche Darmerkrankung, definitionsgemäß gibt es hier keine Ausheilung. Es gibt sicher 2-3\% der Patienten, die lange keinen Schub mehr hatten, einen definierten Zeitraum, in dem von Ausheilung gesprochen werden kann; gibt es aus oben gesagten Gründen nicht.

SpringerMedizin.de/

Expertenrat Gastroenterologie

\section{Einfache Regeln gegen Hypertonie Senkt salzarme Kost den Blutdruck?}

Frage: Bei arterieller Hypertonie wird eine salzarme Kost empfohlen. Diese ist im Alltag nur schwer umzusetzen. Gibt es einfache Tips wie die Patienten versuchen können ihre Salzzufuhr zu verringern bzw. welche Lebensmittel sollten aufgrund Ihres Salzgehalts vermieden werden?

Prof. Dr. Matthias Girndt: Salzarme Kost (ca. 6 g Kochsalz pro Tag) ist bei unseren Ernährungsgewohnheiten gar nicht einfach zu erreichen. Der mittlere Kochsalzkonsum liegt in Deutschland bei etwa $12 \mathrm{~g} / \mathrm{d}$. Es wurde gezeigt, dass die Verminderung der Kochsalzzufuhr um 4-5 g/d den Blutdruck um etwa $5 \mathrm{mmHg}$ senkt, also fast so stark wie ein antihypertensives Medikament (senkt im Schnitt um 8-10 mmHg). Allerdings sind nur etwa $25-30 \%$ der Menschen salzsensitiv, d. h. nur bei diesen tritt der Effekt ein. Dennoch gilt Salzrestriktion mit Recht als eine wichtige antihypertensive Allgemeinmaßnahme.

\section{Die richtige Auswahl}

Einfach Regeln sind: Zurückhaltung bei Fertiggerichten (fast immer stark gesalzen!), beim Mineralwasser auf den $\mathrm{Na}$ triumgehalt achten $(<20 \mathrm{mg} \mathrm{Na} / \mathrm{l})$, hier gibt es enorme Unterschiede. Nicht zusalzen, Gewürzmischungen sparsam einsetzen, salzfreie Einzelgewürze als Geschmacksverstärker nutzen, ggf. Diätsalze (enthalten $\mathrm{KCl}$ ) verwenden (nicht bei Niereninsuffizienz, Vorsicht bei kaliumretinierender Begleitmedikation).

SpringerMedizin.de/

Expertenrat Hypertonie und Kreislauf

\section{www.SpringerMedizin.de}

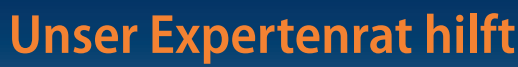
Ihnen weiter!

Sie kommen bei einem Patienten nicht so recht weiter? Suchen eine zweite Meinung bei einem ungewöhnlichen Fall? Bei unseren Expertenräten auf SpringerMedizin.de können Sie Ihre Fragen stellen - und erhalten eine fundierte Antwort. Steuern Sie doch mal das Portlet "Expertenräte" an und schauen Sie, welche Themen dort schon bearbeitet wurden. Die Übersicht der gesamten Expertenräte mitsamt den neuesten Beiträgen und der Möglichkeit, Ihre Frage zu stellen, finden Sie über unsere blaue Navigationsleiste unter dem Button "Community".

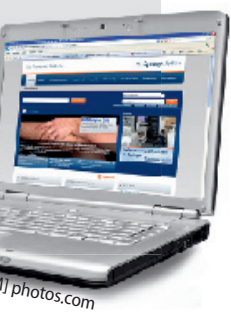

